\title{
PLC $\gamma 2$ regulates osteoclastogenesis via its interaction with ITAM proteins and GAB2
}

\author{
Dailing Mao, ${ }^{1}$ Holly Epple, ${ }^{2}$ Brian Uthgenannt,, ${ }^{1}$ Deborah V. Novack, ${ }^{3}$ and Roberta Faccio' \\ 'Department of Orthopaedic Surgery and Cell Biology, ${ }^{2}$ Department of Medicine and Cell Biology, and ${ }^{3}$ Department of Medicine, \\ Division of Bone and Mineral Diseases, Washington University School of Medicine, St. Louis, Missouri, USA.
}

\begin{abstract}
Excessive bone loss in arthritic diseases is mostly due to abnormal activation of the immune system leading to stimulation of osteoclasts. While phospholipase $\mathrm{C} \gamma(\mathrm{PLC} \gamma)$ isoforms are known modulators of T and B lymphocyte-mediated immune responses, we found that blockade of PLC $\gamma$ enzymatic activity also blocks early osteoclast development and function. Importantly, targeted deletion of Plcg2 in mice led to an osteopetrotic phenotype. $P L C \gamma 2$, independent of PLC $\gamma 1$, was required for receptor activator of NF- $\kappa B$ ligand-induced (RANKL-induced) osteoclastogenesis by differentially regulating nuclear factor of activated T cells c1 (NFATc1), activator protein-1 (AP1), and NF-kB. Specifically, we show that NFATc1 upregulation is dependent on RANKL-mediated phosphorylation of PLC $\gamma 2$ downstream of Dap12/Fc receptor $\gamma(D a p 12 / F c R ~ \gamma)$ receptors and is blocked by the PLC $\gamma$ inhibitor U73122. In contrast, activation of JNK and NF- $K B$ was not affected by U73122 or Dap12/FcR $\gamma$ deletion. Interestingly, we found that in osteoclasts, $\mathrm{PLC} \gamma 2$ formed a complex with the regulatory adapter molecule GAB2, was required for GAB2 phosphorylation, and modulated GAB2 recruitment to RANK. Thus, PLC $\gamma 2$ mediates RANKL-induced osteoclastogenesis and is a potential candidate for antiresorptive therapy.
\end{abstract}

\section{Introduction}

Bone erosion is a major hallmark of rheumatoid arthritis and is solely executed by the bone-resorbing cells, the osteoclasts (OCs) (1). These cells arise from macrophage precursors and differentiate into the mature polykaryon after stimulation with receptor activator of NF- $\kappa \mathrm{B}$ ligand (RANKL) and M-CSF (2). OCs are recruited to sites of inflammation or differentiate at these sites owing to elevated levels of local RANKL secreted by cells in the inflamed tissue. Levels of TNF- $\alpha$, IL-1, and M-CSF are also elevated during inflammatory responses and they collaborate with RANKL to further increase the rate of $\mathrm{OC}$ formation and bone resorption (3-6).

Binding of RANKL to receptor activator of NF-KB (RANK) leads to receptor trimerization, followed by the recruitment of TRAF6, which acts as a key adapter to assemble signaling proteins that direct OC-specific gene expression (2). Both NF-KB and activator protein-1 (AP1) are rapidly induced in response to RANKL, following the activation of IKB kinase $1 / 2$ (IKK1/2) and JNK, respectively $(2,7-9)$. Interfering with IKK1/2 or JNK1 signaling pathways blocks in vitro and in vivo osteoclastogenesis (10-12). Targeted mutagenesis of the p50/p52 components of NF-KB (13, 14) as well as the c-Fos component of AP1 (15) results in osteopetrosis due to a block in osteoclastogenesis. Further, a recent finding indicates that activation of JNK and NF- $\mathrm{KB}$ pathways requires the phosphorylation and the recruitment of the Grb2binding adapter protein, GAB2, to RANK (16). Consistent with these observations, $G A B 2^{-/-}$mice exhibit an osteopetrotic phenotype due to aberrant OC differentiation (16).

In addition to the known roles of AP1 and NF- $\kappa B$ pathways in osteoclastogenesis, recent studies have highlighted the role of the

Nonstandard abbreviations used: AP1, activator protein-1; BMM, BM macrophage; FcR $\gamma$, Fc receptor $\gamma$; IP3, inositol-1,4,5-triphosphate; ITAM, immune receptor tyrosine activation motif; NFATc1, nuclear factor of activated T cells $\mathrm{c} 1$; OB, osteoblast; OC, osteoclast; PLC, phospholipase C; RANK, receptor activator of NF-KB; RANKL, RANK ligand; SFK, Src family kinase; TRAP, tartrate-resistant acid phosphatase.

Conflict of interest: The authors have declared that no conflict of interest exists. Citation for this article: J. Clin. Invest. 116:2869-2879 (2006). doi:10.1172/JCI28775 nuclear factor of activated T cells c1 (NFATc1), a calcineurin- and calcium-regulated transcription factor, as a master transcription factor in this process $(17,18)$. The importance of NFATc1 in OC development has been buttressed by the fact that NFATc1 $1^{-/}$embryonic stem cells cannot differentiate into OCs. Furthermore, overexpression of the WT protein stimulates OC development from embryonic stem cells in a RANKL-independent manner (18). Interestingly, the ability of NFATc1 to mediate osteoclastogenesis is strictly connected to the c-Jun pathway (19). Transgenic mice expressing a dominant negative form of c-Jun have osteopetrosis due to defective osteoclastogenesis that cannot be rescued by NFATc1 overexpression in OCs (19).

Immune receptor tyrosine activation motifs (ITAMs) are important signaling components for a number of receptors in the immune system, and they have been recently identified as costimulatory signals in RANKL-induced osteoclastogenesis (20). The 2 ITAMcontaining adapters, Dap12 and the $\gamma$ chain of Fc receptor $(\mathrm{FcR} \gamma)$, have a profound impact on OCs by modulating RANKL-mediated calcium influx and NFATc1 expression. In humans, mutation in the Dap12 gene is responsible for Nasu-Hakola disease, characterized by multiple bone cysts associated with a unique form of neurodegeneration (21). Deletion of Dap12 and $F c R \gamma\left(D a p 12^{-/-} F c R \gamma^{-/}\right)$, in mice, leads to severe osteopetrosis due to defective OC development (20-22). In vitro triggering of either receptor by crosslinking with an antibody accelerates RANKL-induced OC differentiation (23). However, in the absence of RANKL, these receptor-mediated signals alone are not sufficient to induce OC differentiation.

The phospholipase C (PLC) family regulates PKC activation, intracellular calcium levels, and NFAT expression in hematopoietic systems in response to the stimulation of immune receptors (24-27). PLC cleaves the membrane phospholipid phosphatidyl inositol-4,5-biphosphate (PIP2) into the second messenger molecules inositol-1,4,5-triphosphate (IP3) and diacylglycerol (DAG). IP3 directly increases intracellular calcium levels by inducing the release of endoplasmic reticulum calcium stores, while DAG activates $\mathrm{PKC}$ at the plasma membrane. 
A

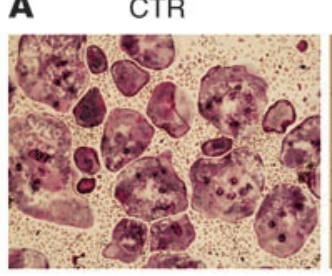

B

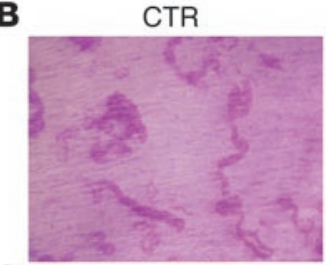

C

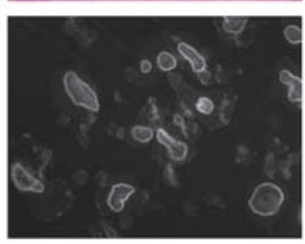

$+\mathrm{U} 73122$
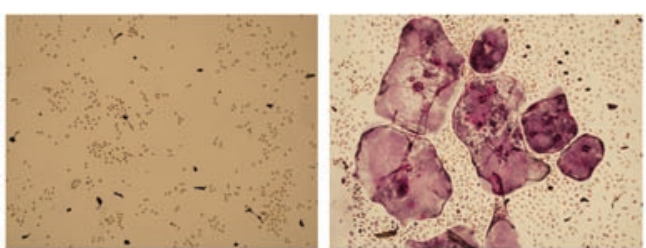

$+\mathrm{U} 73122$
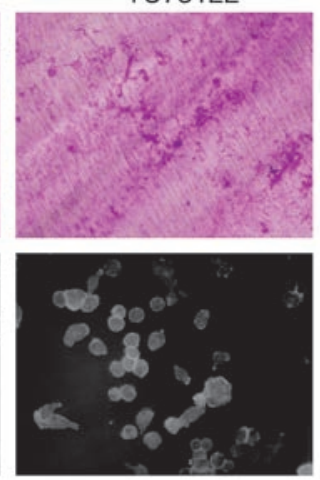

\section{Figure 1}

PLC $\gamma$ inhibition blocks osteoclastogenesis and actin ring formation. (A) WT OCs were generated with RANKL (100 $\mathrm{ng} / \mathrm{ml})$ and M-CSF $(10 \mathrm{ng} / \mathrm{ml})$ in the presence of the PLC inhibitor U73122 (5 $\mu \mathrm{M})$ for 4 days on plastic. In some wells, the media with the inhibitor was replaced with fresh osteoclastogenic media, and cells were allowed to differentiate for 4 more days (Withdrawal U73122). (B) WT OCs were grown on dentin with or without U73122 for 10 days. Cells were then removed and pits stained with hematoxylin red (Magnification, $\times 200$ ). CTR, control. (C) WT OCs were generated on dentin in the absence of the inhibitor, then treated with vehicle or U73122 for 1 hour. Cells were fixed and actin stained using FITC-phalloidin (Magnification, ×200).
The PLC $\gamma$ family consists of 2 members, PLC $\gamma 1$ and PLC $\gamma 2$ (24-26). Both isoforms require phosphorylation on specific tyrosine residues for their catalytic activity. In addition, they contain $2 \mathrm{SH} 2$ and $1 \mathrm{SH} 3$ domains through which they can interact with other signaling proteins, suggesting adapter function. PLC $\gamma 1$ is widely distributed, while PLC $\gamma 2$ is primarily limited to cells of hematopoietic lineage (24). Mice deficient in PLC $\gamma 1$ develop normally to embryonic day 8.5 but die soon after due to undefined defects in growth and development (28). The disruption of PLC $\gamma 2$ in mice does not result in embryonic lethality, but animals show internal bleeding, decreased B cell number and function, defective platelet aggregation to collagen, and a block in Fc receptor-mediated responses in mast cells and NK cells (29-31).

Although NF-кB, AP1, and NFATc1 are all critical for RANKLinduced osteoclastogenesis $(9,18,32)$, due to their ubiquitous expression, selective therapies targeting these signaling pathways in OCs are not yet in clinical use. In this study, we show that targeted deletion of $\mathrm{Plcg} 2$ in mice results in an in vivo osteopetrotic phenotype, independent of PLC $\gamma 1$. In vitro, Plcg2-null cells fail to form multinucleated OCs and exhibit decreased RANKL-mediated NFATc1 expression; impaired JNK, c-Jun, and IкB $\alpha$ phosphorylation; and thus defective AP1 and NF- $\mathrm{\kappa B}$ activation. Our data reveal a role for PLC $\gamma 2$ in bone homeostasis apart from its contribution to $\mathrm{B}$ cell development. The combined effect of PLC $\gamma 2$ on lymphocyte and OC differentiation could represent a novel target for the treatment of inflammatory osteolytic diseases.

\section{Results}

Inhibition of PLC $\gamma$ enzymatic activity blocks OC differentiation and disrupts actin ring formation. PLC $\gamma$ isoforms are known activators of the NFAT family of transcription factors in lymphoid cells, downstream of immune receptor signaling via ITAMs (33). Since NFAT and ITAMs are both critical regulators of OC differentiation and cells lacking the ITAM-containing adapters Dap12/FcR $\gamma$ display severe osteopetrosis (20), we asked whether PLC $\gamma$ family members were also required for efficient $\mathrm{OC}$ differentiation and function. We therefore tested the capacity of the PLC $\gamma$ inhibitor U73122 to block OC formation and bone resorption. Addition of U73122 to the culture media blocked OC development, as demonstrated by the absence of tartrate-resistant acid phosphatase-positive (TRAPpositive) cells, compared with the numerous multinucleated OCs obtained in wells cultured only with RANKL and M-CSF (Figure 1A). Replacing the media with fresh osteoclastogenic media lacking the inhibitor restored the ability of the cells to differentiate, indicating that the compound was not toxic to the cells (Figure 1A). Culture of OCs on dentin in the presence of U73122 blocked bone resorption (Figure 1B). Furthermore, addition of U73122 to mature resorbing cells rapidly disrupted the organization of the actin rings, indicating that this compound can block bone resorption independent of its capacity to affect differentiation (Figure 1C).

$P L C \gamma 2$ regulates bone mass in vivo. To determine whether PLC $\gamma 1$, $\mathrm{PLC} \gamma 2$, or both are required for OC formation, we turned to the analysis of mice lacking these proteins. $P \operatorname{lcg} 1^{-/-}$mice die in utero by day 9 , rendering the analysis of their bone phenotype difficult. Plcg2 $2^{-/}$mice, however, are viable. Thus, femurs from 4-month-old sex-matched WT and Plcg2-/- mice were subjected to histological examination (Figure 2, A-E) and $\mu \mathrm{CT}$ analysis (Figure 2, F-I). Plcg2 $2^{--}$mice had a more than 3 -fold increase in the percentage of trabecular bone volume versus total bone volume compared with their WT counterparts, as determined by histological examination (Figure $2 \mathrm{~B}$ ) and as shown in $\mu \mathrm{CT}$ reconstructed $3 \mathrm{D}$ images (Figure $2 \mathrm{~F}$ ). Histomorphometric analysis also indicated that the number of osteoblasts (OBs) per bone perimeter was similar in WT and $\mathrm{Plcg} 2^{-/-}$mice (Figure 2C), while the number of OCs per bone perimeter and the OC surface were significantly decreased in the null animals (Figure 2, D and E). The number of the trabeculae (Figure $2 \mathrm{G}$ ) and their thickness (Figure $2 \mathrm{H}$ ) were significantly higher in the $\mathrm{Plcg}^{-/-}$compared with WT mice, while the space between the trabeculae was smaller (Figure 2I). Thus, PLC $\gamma 2$ regulates bone mass in vivo.

$P L C \gamma 2$ is required for OC differentiation. To determine whether PLC $\gamma 2$ modulates the capacity of OBs to form bone or to promote OC differentiation, primary OBs were isolated from newborn mice, and their capacity to mineralize extracellular matrix or induce osteo- 

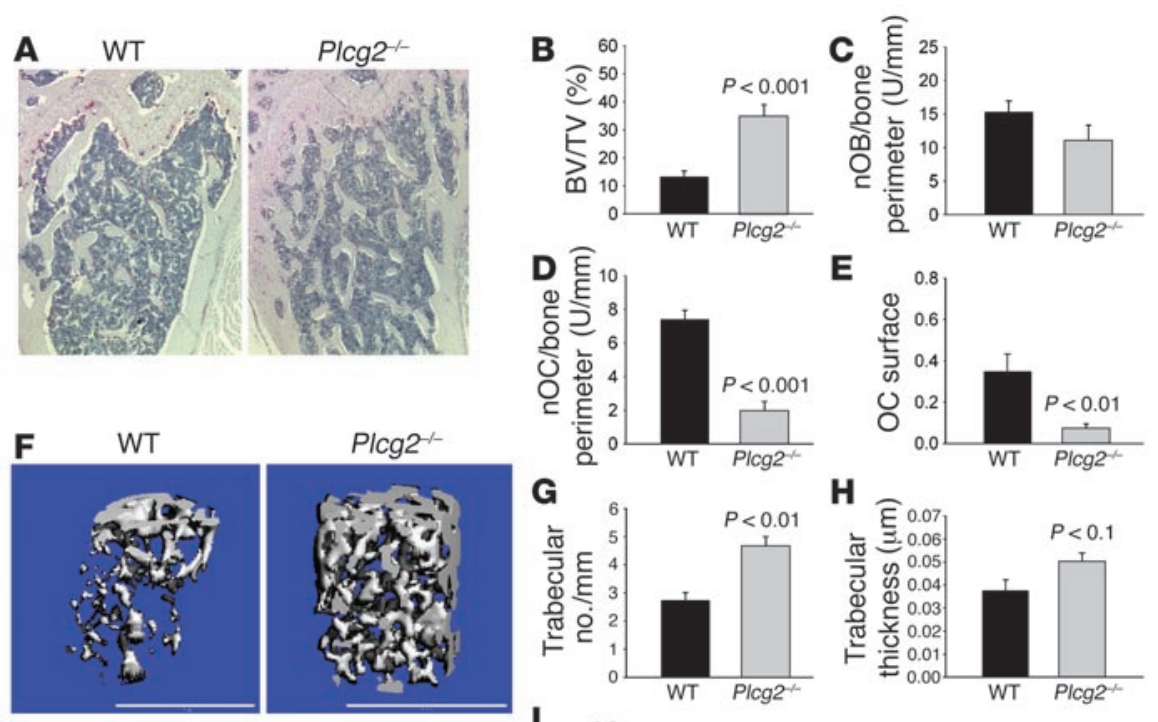

\section{E}
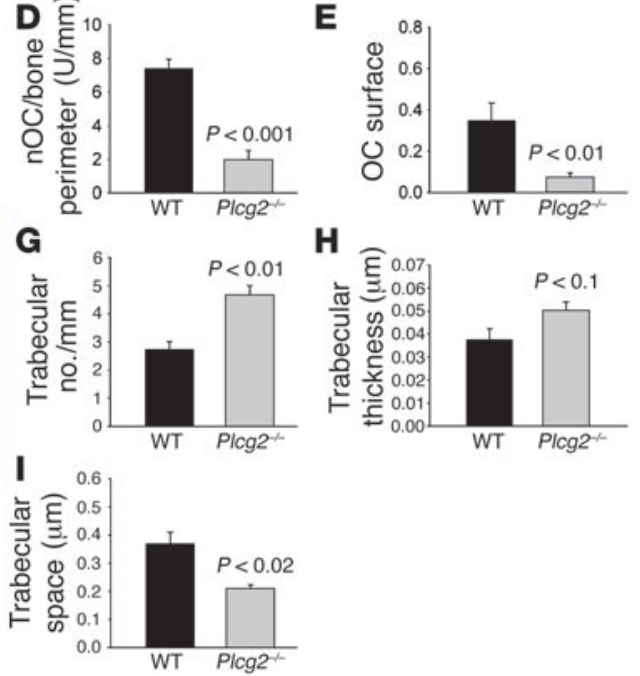

\section{Figure 2}

Osteopetrosis in mice lacking PLC $\gamma 2$. (A) TRAP staining of decalcified histological sections of WT and $\mathrm{Plcg}^{-/-}$proximal femurs. Magnification, $\times 40$. (B-E) Quantitative analysis of bone parameters from histological sections of WT and Plcg2 $^{-/-}$bone femurs $(n=8)$ showing: percentage of bone volume versus total bone volume (BV/TV) (B); number of OBs (nOB) per bone perimeter (C); number of OCs (nOC) per bone perimeter (D); fraction of trabecular surface covered by OCs (E). (F) 3D reconstitution of $\mu \mathrm{CT}$ scans of WT and Plcg2 $2^{-/-}$femurs. (G-I) 3D trabecular quantitative parameters of bone structure $(n=6)$. Graphs show mean \pm SEM, with significant differences compared with WT indicated. clastogenesis in a coculture system was examined. Plcg2-/- OBs were indistinguishable from their WT counterparts in their ability to form bone nodules in response to $\beta$-glycerolphosphate (WT, $14 \pm 2.08$ nodules/well versus $P l c g 2^{-/-}, 14.6 \pm 1.65$ nodules/well) (Figure 3A). This finding was also supported by the in vivo analysis of bone mineral apposition determined by calcein double-labeling (Figure 3B), which revealed no significant difference in bone formation rates (BFRs) and mineral apposition rates (MARs) between WT (BFR, $0.8938 \pm 0.1042 \mu \mathrm{m}^{3} / \mu \mathrm{m}^{2} / \mathrm{d} ;$ MAR, $0.5927 \pm 0.0219 \mu \mathrm{m} / \mathrm{d}$ ) and Plcg2 $2^{--}$animals (BFR, $0.8450 \pm 0.1629 \mu \mathrm{m}^{3} / \mu \mathrm{m}^{2} / \mathrm{d} ; \mathrm{MAR}$, $0.4015 \pm 0.0876 \mu \mathrm{m} / \mathrm{d}$ ). To determine whether $\mathrm{Plcg} 2^{-/-}$OBs are capable of supporting OC differentiation, BM macrophages (BMMs) from WT mice were cocultured with either WT or Plcg2-/- OBs in the presence of 1,25 vitamin $\mathrm{D}_{3}\left(1,25\right.$ Vit $\left.\mathrm{D}_{3}\right)$. Cultures were stained for TRAP to identify OCs. WT BMMs became TRAP-positive, multinucleated cells when cultured with WT OBs (OCs, $9 \pm 1.07 /$ well) or null OBs (OCs, $7 \pm 0.44 /$ well), indicating that the expression of $\mathrm{PLC} \gamma 2$ in OBs is not required to support osteoclastogenesis. However, in a parallel experiment, BMMs from $\mathrm{Plcg}^{-1-}$ mice failed to form mature OCs in the coculture system, independent of the source of the OBs (Figure 3C). These results suggest that the increased in vivo bone mass observed in $\mathrm{Plcg}^{-\gamma_{-}}$mice was not due to aberrant OB activity but was dependent on an intrinsic defect in the OC lineage. We therefore cultured primary BMMs with M-CSF and RANKL for 5 days, in the absence of OBs or stromal cells. The osteoclastogenic response of $\mathrm{Plcg}^{-2^{--}}$BMMs to RANKL and their capacity to resorb bone were arrested (Figure 3D; WT OCs, $33.2 \pm 1.74$ /well versus Plcg2 $2^{-/}$OCs, not detected). Increasing the concentration of M-CSF from 10 to $100 \mathrm{ng} / \mathrm{ml}$, a condition that partially rescued the osteoclastogenesis in cells lacking the ITAM-bearing adapter Dap12 (34), did not enhance Plcg2 $2^{--}$OC differentiation (data not shown).

To determine which stage of OC differentiation is PLC $\gamma 2$ dependent, the levels of early osteoclastogenic markers TRAP, cathepsin K, NFATc1, and calcitonin receptor, a late OC marker, were determined by real-time PCR analysis in BMMs cultured with RANKL
$(100 \mathrm{ng} / \mathrm{ml})$ and M-CSF $(10 \mathrm{ng} / \mathrm{ml})$ up to 4 days. The expression of TRAP, cathepsin K, and NFATc1 increased within 24-48 hours after RANKL stimulation in WT but not in $\mathrm{Plcg}^{-2^{--}}$cells (not shown). On day 4, the levels of these genes were maximal in WT cells but dramatically lower in the null cells (Figure 4, A-D). Similarly, calcitonin receptor was detected after 4 days of treatment with RANKL only in WT OCs, suggesting that Plcg2-/- cells have an early block of OC development. Confirming mRNA expression, protein levels of NFATc1 were significantly higher in total cell lysates of day 4 WT OCs than in null cells, both under basal conditions and following 60 minutes of RANKL stimulation (Figure $4 \mathrm{E})$. Together, these data indicate that PLC $\gamma 2$ is required at early stages of OC development.

$P L C \gamma 2$ is required for efficient RANKL-induced activation of AP1 and $N F-\kappa B$ in developing OCs. To further understand whether PLC $\gamma 2$ is a downstream mediator of RANKL, M-CSF, or both during OC differentiation, we analyzed MAPK activation by BMMs in culture with M-CSF for 3 days. Activation of ERK and JNK in response to M-CSF occurred normally in BMMs lacking PLCy2 (Figure 5A). Similarly, ERK was normally phosphorylated in the null cells in response to RANKL (Figure 5B). In contrast, RANKL-mediated JNK phosphorylation was retarded and reduced in the absence of PLC 22 (Figure 5B), and activation of c-Jun (by phosphorylation) was also decreased in both the cytoplasm and the nucleus (Figure 5, B and C). This result is particularly compelling, since JNK/c-Jun activates the AP1 complex, and inhibition of JNK activity blocks RANKL-induced OC development (19). Thus, to determine whether the decreased phosphorylation of JNK observed in Plcg2 $2^{-/}$BMMs stimulated with RANKL could result in deficient AP1 activation, nuclear extracts from WT and Plcg2-- cells were subjected to EMSA analysis following RANKL stimulation. The activation of AP1 was abolished in RANKL-stimulated Plcg2-/BMMs (Figure 5D, top panel).

Since NF-KB is the other major signaling pathway required for efficient osteoclastogenesis, and PLC $\gamma$ isoforms have been shown 
A
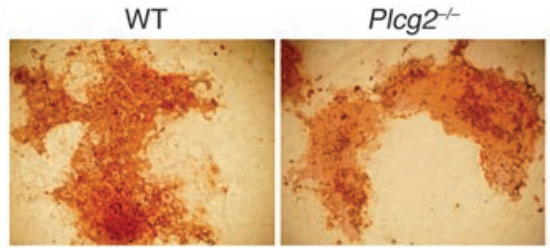

C

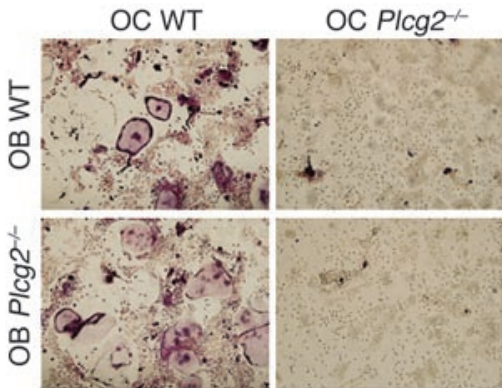

B

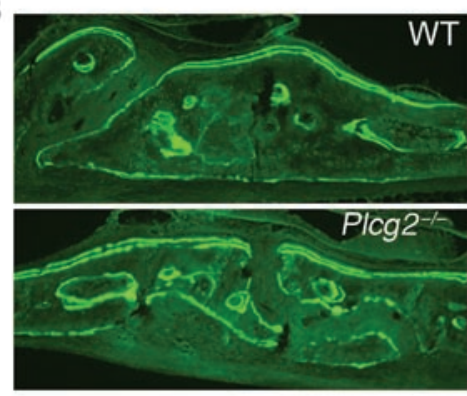

D
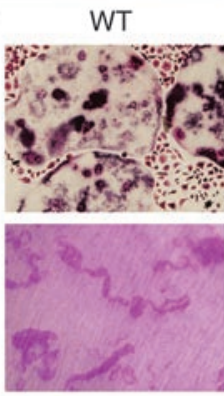

$\mathbf{E}$

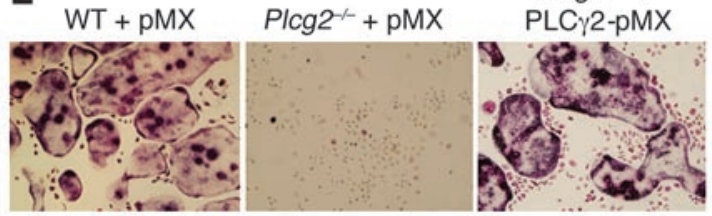

$\mathrm{Plcg}^{-}+$ PLC $2-p M X$

Figure 3

PLC $\gamma 2$ is required for osteoclastogenesis. (A) Bone nodule formation in WT and $\mathrm{Plcg}^{-/-}$OBs cultured with ascorbic acid and $\beta$-glycerolphosphate. (B) Double labeling of calvarial bones showing the degree of bone formation (BFR) of WT and $P / \mathrm{cg}^{-/-}$mice injected on day 0 and day 7 with calcein (WT BFR, $0.8938 \pm 0.1042 \mu \mathrm{m}^{3} / \mathrm{um}^{2} / \mathrm{d}$; WT mineral apposition rate [MAR], $0.5927 \pm 0.0219 \mu \mathrm{m} / \mathrm{d}$;

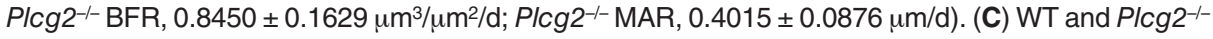
BMMs cultured with WT and Plcg2-l- OBs in the presence of $10^{-8} \mathrm{M} \mathrm{1,25} \mathrm{Vit} \mathrm{D}_{3}$. After 14 days, cells were fixed and TRAP stained to detect the presence of multinucleated OCs. (D) TRAP-stained OCs generated with RANKL (100 ng/ml) and M-CSF (10 ng/ml) for 5 days (top panel) and bone resorptive pits generated from OCs plated on dentin for 7 days. Pits were stained with hematoxylin red. (E) WT and $\mathrm{Plcg}^{-1-}$ BMMs retrovirally transduced with vector alone $(\mathrm{pMX})$ or with Flag-tagged PLC $\gamma 2$ were allowed to differentiate in osteoclastogenic media for 5 days, then stained for TRAP. Magnification, $\times 200$ (A, C-E); ×100 (B).

to modulate $\mathrm{T}$ cell and $\mathrm{B}$ cell receptor-mediated NF-KB activation, we hypothesized that this pathway could also be regulated by PLC $\gamma 2$ in OCs. Nuclear extracts from WT and Plcg2- BMMs stimulated with RANKL were subjected to EMSA analysis, and data show that in the null cells, activation of NF-KB was virtually absent (Figure 5D, bottom panel). In agreement with this result, IкB $\alpha$ phosphorylation and nuclear translocation of $\mathrm{p} 65$ were also decreased in Plcg2 $2^{--}$BMMs (Figure 5, B and C). Collectively, these data suggest that PLC $\gamma 2$ plays a central role in RANKL-mediated osteoclastogenesis by controlling activation of AP1 and NF- $\mathrm{\kappa B}$ and upregulation of NFATc1.

PLC 22 is phosphorylated by RANKL via Dap12-mediated costimulatory signals in a Srcfamily kinase-dependent manner. Having shown that PLC $\gamma 2$ is required for the activation of osteoclastogenic signaling pathways downstream of RANKL, we turned to the mechanism by which PLC $\gamma 2$ itself is activated in OCs. In immune cells, $\mathrm{PLC} \gamma$ isoforms are tyrosine phosphorylated in a Src family kinase-dependent (SFK-dependent) manner (24). Incubation of WT cells with the SFK inhibitor PP2 $(5 \mu \mathrm{M})$ completely blocked RANKL-mediated PLC $\gamma 2$ phosphorylation and NFATc1 upregulation (Figure 6A), demonstrating a concordant dependence on SFK in OC lineage cells.

Plcg $2^{-1-}$

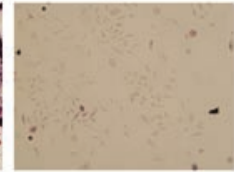

$15-x=3$
To determine whether lack of PLC $\gamma 2$ was abrogating the activation of PLC $\gamma 1$ in response to RANKL and M-CSF, WT and $P \operatorname{lcg} 2^{--}$OC precursors (BMMs grown in RANKL-containing media for 2 days) were stimulated with RANKL or M-CSF. Interestingly, RANKL-mediated PLC $\gamma 1$ phosphorylation was barely measurable in both WT cells and cells lacking PLC $\gamma 2$ (Figure 6B) despite detectable levels of PLC $\gamma 1$ protein in both cell types. In contrast, a strong PLC $\gamma 1$ phosphorylation signal was observed after M-CSF stimulation in WT and Plcg2 $2^{--}$cells (Figure 6C).

Since costimulatory signals are required for RANKL-mediated calcium oscillation and NFATc1 expression, we determined whether PLC $\gamma 2$ phosphorylation was dependent on the RANKL costimulatory receptors Dap12 and FcR $\gamma$. We found that PLC $\gamma 2$ phosphorylation and NFATc1 nuclear localization were decreased in Dap12-/ $\mathrm{FcR} \gamma^{-/}$cells treated with RANKL (Figure 6, E and F). Reexpression of Dap12, but not empty vector, in the double null OCs (Figure 6D) completely restored PLC $\gamma 2$ phosphorylation (Figure 6E) and expression of NFATc1 in the nucleus (Figure 6F). Thus, PLC $\gamma 2$ is phosphorylated downstream of Dap12 in an SFK-dependent manner, following RANKL stimulation.

$P L C \gamma 2$ catalytic activity is required for NFATc1 upregulation but not for JNK and I $\kappa \alpha$ phosphorylation. Our data indicate that PLC $\gamma 2$ regulates NFATc1 and activates JNK and IKB $\alpha$. In contrast, Dap12/FcR $\gamma$ modulates NFATc1 (Figure 6F) but not JNK (Figure $6 \mathrm{E})$ or IкB $\alpha(20)$. Thus, we hypothesized that PLC $\gamma 2$ regulates JNK and IKB $\alpha$ independent of Dap12/FcR $\gamma$. To determine whether PLC $\gamma 2$ catalytic activity is required for JNK and I $\mathrm{K} B \alpha$ phosphorylation, WT OC precursors, cultured in the presence of the PLC inhibitor U73122 $(5 \mu \mathrm{M})$, were stimulated with RANKL for 0 to 60 minutes (Figure 7A). While dampening NFATc1 upregulation, the inhibitor had no effect on $\mathrm{p}$-JNK and $\mathrm{p}$-I $\mathrm{KB} \alpha$, suggesting that the catalytic activity of PLC $\gamma 2$ is not required for phosphorylation of JNK and IкB $\alpha$.

Mutations of 2 histidine residues, conserved among all PLC family members, have been shown to inhibit the enzymatic activity of PLC $\gamma 1$ by $90 \%-95 \%$ in vitro (35). Thus, we generated a PLC $\gamma 2$ catalytic inactive construct carrying the double histidine 327/372 to phenylalanine mutation (PLC $\gamma 2 \mathrm{H} / \mathrm{F}$ ). This mutant was proven to be lipase inactive compared with WT full-length PLC $\gamma 2$ in 293 cells (data not shown). Plcg2 $2^{-/}$cells transduced with PLC $\gamma 2$ $\mathrm{H} / \mathrm{F}$ were incapable of generating mature, TRAP-positive OCs compared with cells expressing WT PLC $\gamma 2$ (Figure 7B) or of promoting NFATc1 upregulation (Figure $7 \mathrm{C}$ ). In contrast, the ability of PLC $\gamma 2 \mathrm{H} / \mathrm{F}$ to mediate JNK and IкB $\alpha$ phosphorylation was 
A

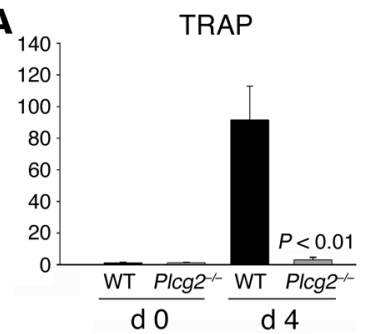

B

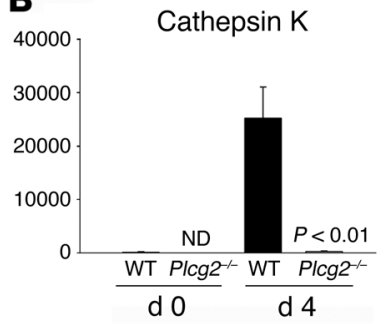

C

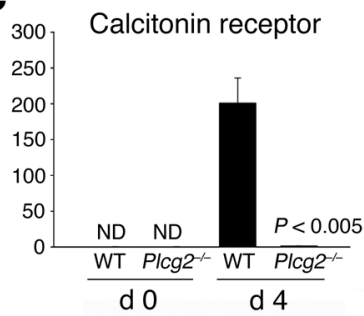

D

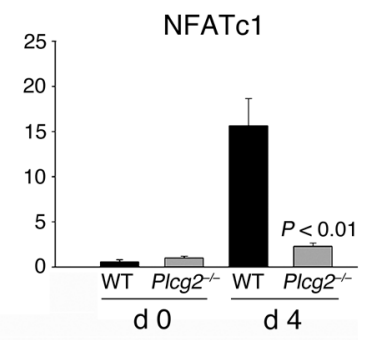

$\mathbf{E}$
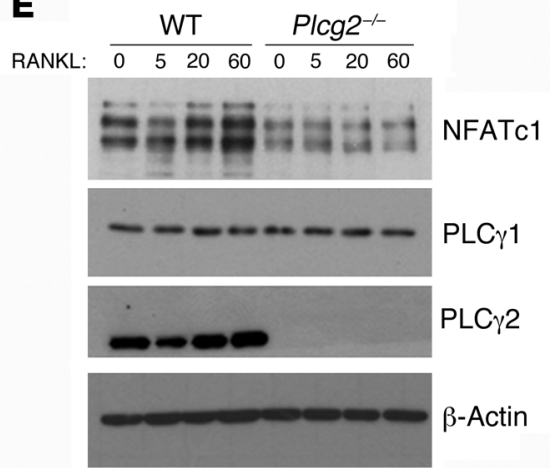

\section{Figure 4}

PLC $\gamma 2$ regulates NFATc1 levels and the expression of early osteoclastogenic genes. (A-D) Real-time PCR analysis of osteoclastogenic markers in WT and $\mathrm{Plcg}^{-/-}$cells in culture with M-CSF alone (d0) or with RANKL (100 $\mathrm{ng} / \mathrm{ml})$ plus M-CSF (10 ng/ml) for 4 days (d4). Data are normalized relative to $\beta$-actin. ND, not detected. Significant differences compared with WT are shown. (E) Expression levels of NFATc1 protein in total cell lysates from day 4 WT and lcg $^{-1-}$ OCs, starved for 6 hours and stimulated with RANKL for $0,5,20$, and 60 minutes. PLC $\gamma 1$ and PLC $\gamma 2$ levels are shown. $\beta$-Actin served as loading control.

JNK but not NFATc1 (16). Furthermore, GAB2 has been shown to bind PLC $\gamma 2$ in mast cells (36). To determine whether PLC $\gamma 2$ binds to GAB2 in OCs, we reciprocally immunoprecipitated GAB2 and PLC 2 and found that the 2 molecules associated (Figure 7E). Immunoprecipitation with IgG control antibodies revealed the absence of nonspecific bands (data not shown). To further determine whether PLC $\gamma 2$ modulates GAB2 activation or its recruitment to RANK, WT and Plcg2-/- BMMs, treated with RANKL, were immunoprecipitated with an anti-GAB2 $\mathrm{Ab}$ (Figure 7F) or with rabbit IgG control $\mathrm{Ab}$ (data not shown), followed by immunoblotting with phosphotyrosine (4G10) and RANK. Results showed that PLC $\gamma 2$ modulates GAB2 phosphorylation and its association with RANK, suggesting that this may be the mechanism by which PLC $\gamma 2$ controls AP1 and NF-kB activation.

$T N F-\alpha$ cannot reverse the arrested capacity of PLC 2 2-null cells to became OCs. The data presented above show that PLC $\gamma 2$ is central in RANKL signaling. In OCs, TNF- $\alpha$ appears to be the dominant cytokine mediating inflammatory osteolysis and augments the indistinguishable from that of WT PLC $\gamma 2$ (Figure 7D). These data indicate that PLC $\gamma 2$ can modulate RANK-mediated signaling independent of its catalytic activity by acting as an adapter molecule. One potential interacting protein is GAB2, which associates with RANK and mediates RANK-induced activation of NF- $\kappa B$ and
A M-CSF

\section{Figure 5}

PLC $\gamma 2$ modulates RANKL-mediated signaling. (A) Activation of ERK and JNK in WT and $\mathrm{Plcg}^{-/-}$BMMs in response to M-CSF (100 ng/ml). PLC $\gamma 1$ and PLC $\gamma 2$ levels are shown. (B) Western blot analysis of phospho-ERK, phospho-JNK, phospho-C-Jun, and phospho- $\mathrm{I}_{\mathrm{K}} \mathrm{B} \alpha$ in total cell lysates from WT and Plcg2 ${ }^{-/-}$ BMMs stimulated with RANKL (100 ng/ $\mathrm{ml}$ ) for the indicated times. $\beta$-Actin blots served as loading control for $\mathbf{A}$ and $\mathbf{B}$. (C) Nuclear levels of phospho-C-Jun and p65 in nuclear extracts from WT and Plcg2 $^{-/-}$BMMs stimulated with RANKL. (D) The same samples used in $\mathbf{C}$ were subjected to AP1 and NF-кB nonradioactive EMSA analysis. To control for binding specificity, a concentration of unlabeled oligonucleotides (U.O.) 200fold greater than that recommended by the manufacturer was added to nuclear extracts from WT cells stimulated with RANKL for 60 minutes. SP1 served as loading control for $\mathbf{C}$ and $\mathbf{D}$.
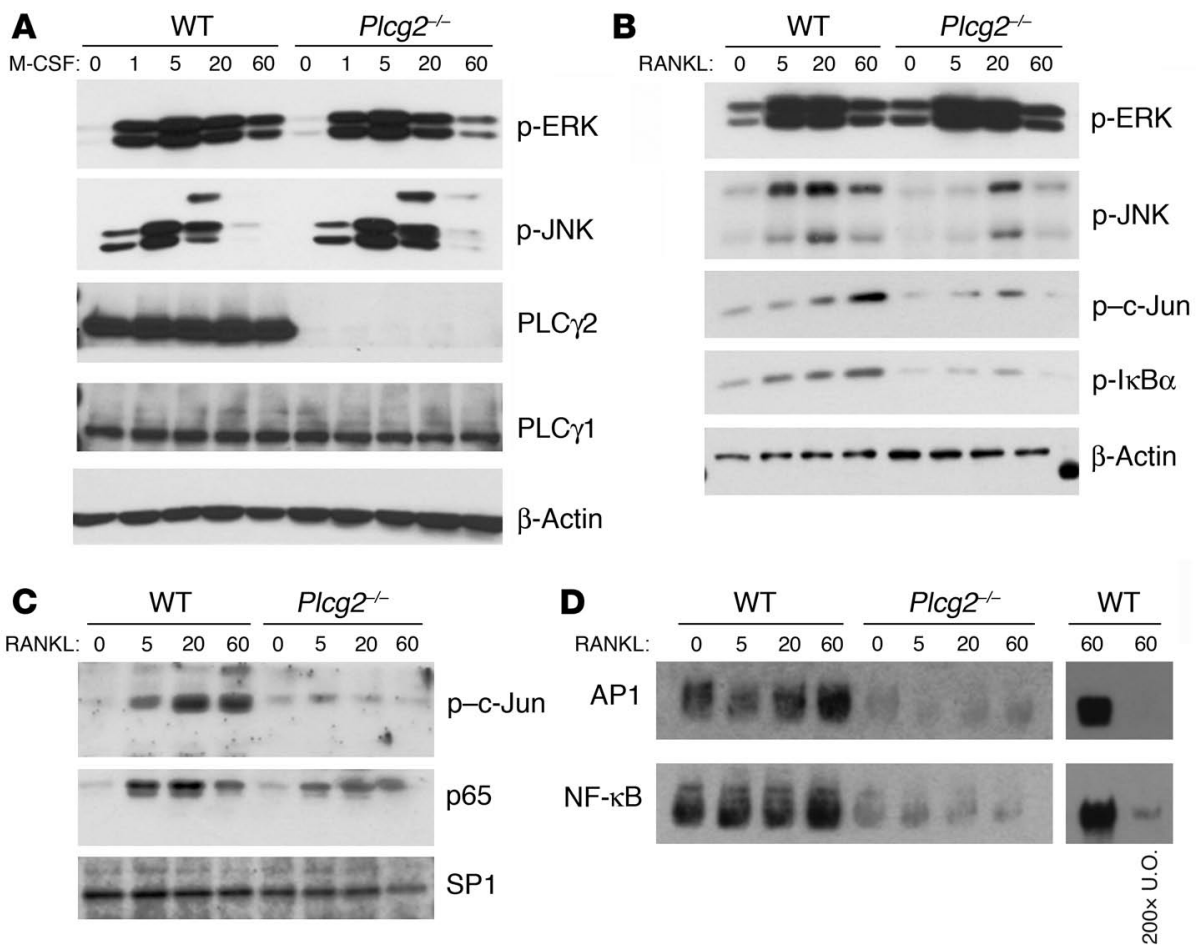

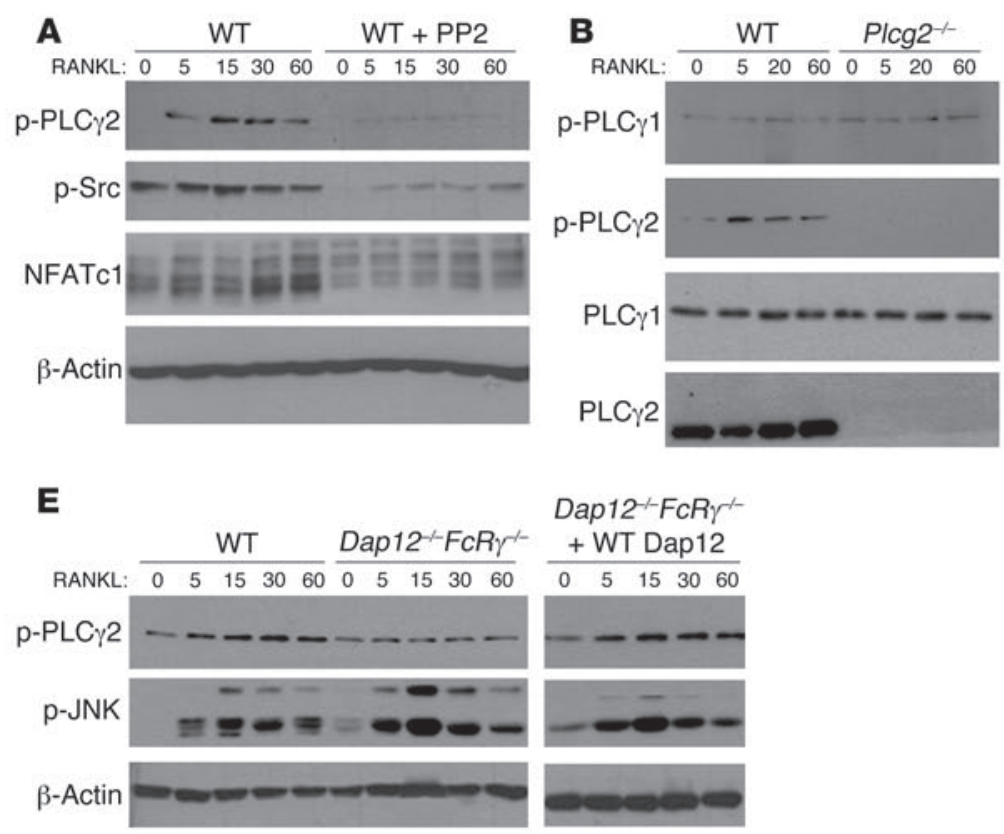

Dap 12--FcRr-

$\frac{\text { +WT Dap } 12}{0 \quad 5153060}$

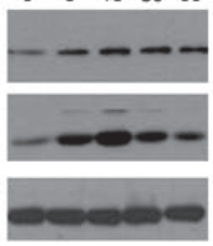

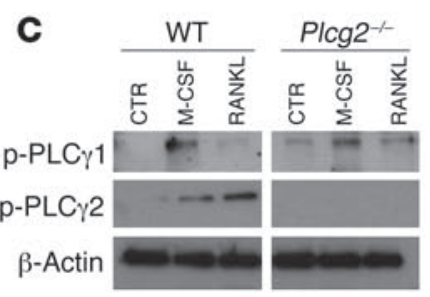

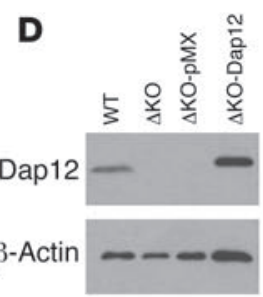

\section{F}

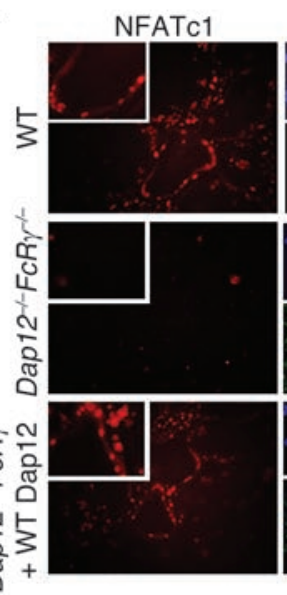

$\mathrm{DAPI}+$ actin

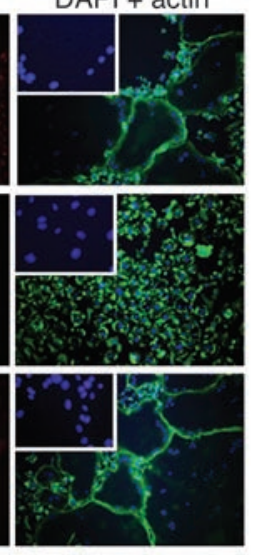

\section{Figure 6}

PLC $\gamma 2$ is activated by RANKL via Dap12/FcR in an SFK-dependent manner. (A) WT OC precursors (preOCs; BMMs grown in RANKL-containing media for 2 days) cultured with the SFK inhibitor PP2 $(5 \mu \mathrm{M})$ or vehicle (DMSO) were stimulated with RANKL and subjected to Western blot analysis to detect phosphorylated levels of PLC $\gamma 2$, Src, and NFATc1. $\beta$-Actin served as control. (B) PLC $\gamma 1$ and PLC $\gamma 2$ phosphorylation in response to RANKL were measured by Western blot analysis in WT and Plcg2-/- preOCs. PLC $\gamma 1$ and PLC $\gamma 2$ levels are shown. (C) PLC $\gamma 1$ and PLC $\gamma 2$ phosphorylation in response to 5 minutes of treatment with either M-CSF or RANKL in WT and Plcg $2^{-/-}$preOCs. $\beta$-Actin served as control. (D) Expres-

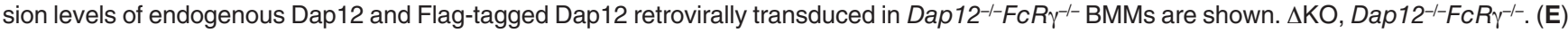
PLC 22 phosphorylation was measured by Western blot analysis in WT, Dap $12^{-1-} F c R \gamma^{-1-}$, or Dap $12^{-1-} F_{C} R \gamma^{-1-}$ preOCs reconstituted with WT Dap12 stimulated with RANKL for the indicated times. Phospho-JNK is also shown. $\beta$-Actin served as loading control. (F) Nuclear localization of NFATc1 in WT and Dap12--FcR $\gamma^{-1-}$ OCs retrovirally transduced with pMX or Flag-tagged Dap12 is shown in red (left panels). Actin staining is shown in green, and nuclei, stained with DAPI, are shown in blue (right panels) (objective, $\times 20)$. Enlarged images (2.5-fold) show nuclear localization of NFATc1 (red) and nuclei stained with DAPI (blue) of representative cells located in the center of the photographed field.

osteoclastogenic potential of RANKL-exposed OC precursors (3). Due to the capacity of TNF- $\alpha$ to phosphorylate JNK and $\mathrm{I} \kappa \mathrm{B} \alpha$ in BMMs and OCs (37), we tested its effect in modulating the above-mentioned signaling pathways in cells lacking PLC $\gamma 2$. TNF- $\alpha$-mediated phosphorylation of JNK and I $\kappa \mathrm{B} \alpha$ occurred similarly in WT and null cells (Figure 8A). Furthermore, TNF- $\alpha$ was not capable of inducing PLC $\gamma 2$ phosphorylation (Figure 8A), suggesting that the mechanism by which TNF- $\alpha$ regulates JNK and I $\kappa \mathrm{B} \alpha$ is PLC $\gamma 2$ independent. This result prompted us to determine whether TNF- $\alpha$ could rescue the osteoclastogenic defect of Plcg2 $2^{-/}$cells. Interestingly, while TNF- $\alpha$ augmented RANKL-mediated osteoclastogenesis of WT cells (WT OCs without TNF- $\alpha$, $19.3 \pm 1.5$ /well; WT OCs with TNF- $\alpha, 45.3 \pm 1.99$ /well), the cytokine did not have any effect on the null cells, which remained blocked at the early stage of OC development (Plcg2-/- OCs, not detected) (Figure 8B). Consistent with this finding, TNF- $\alpha$, compared with RANKL, was not capable of promoting NFATc1 upregulation in WT or in PLC 2 2-null cells (Figure 8C).

\section{Discussion}

The interaction between the immune and skeletal systems is a significant cause of pathological bone loss in rheumatoid arthritis, periodontal diseases, and tumor-associated bone metastasis (38).
This exaggerated bone loss is most frequently due to abnormal activation of the immune system, leading to hyperstimulation and differentiation of the OCs $(2,39)$. The identification of signaling pathways or molecules common to the immune and bone systems could help in designing therapies for the variety of diseases that affect both bone and immunity. Recently, an activating point mutation in the murine PLC $\gamma 2$ gene has been shown to lead to severe spontaneous inflammation and autoimmunity, mediated by B cells and non-B and non-T hematopoietic cells (40). The chronic inflammation resulted in severe arthritis of the small joints, leading to phalangeal erosion (40). This observation suggested that the activating mutation in PLC $\gamma 2$ gene could also be responsible for the hyperactivation and recruitment of the OCs at the inflammatory sites.

In this study we have identified what we believe to be a novel role for the immunomodulatory protein PLC $\gamma 2$ as a key factor in the regulation of bone homeostasis. Targeted deletion of Plcg2 led to increased bone mass due to blockade of OC differentiation in unmanipulated mice, with decreased RANKL-mediated signaling to AP1 and NF- $\mathrm{BB}$ and defective upregulation of NFATc1, independent of PLC $\gamma 1$. Despite the presence of normal levels of PLC $\gamma 1$ in the PLC $\gamma 2$ null cells, PLC $\gamma 1$ could not compensate for the lack of its cognate isoform. Furthermore, we found that PLC $\gamma 2$, and not PLC $\gamma 1$, was 

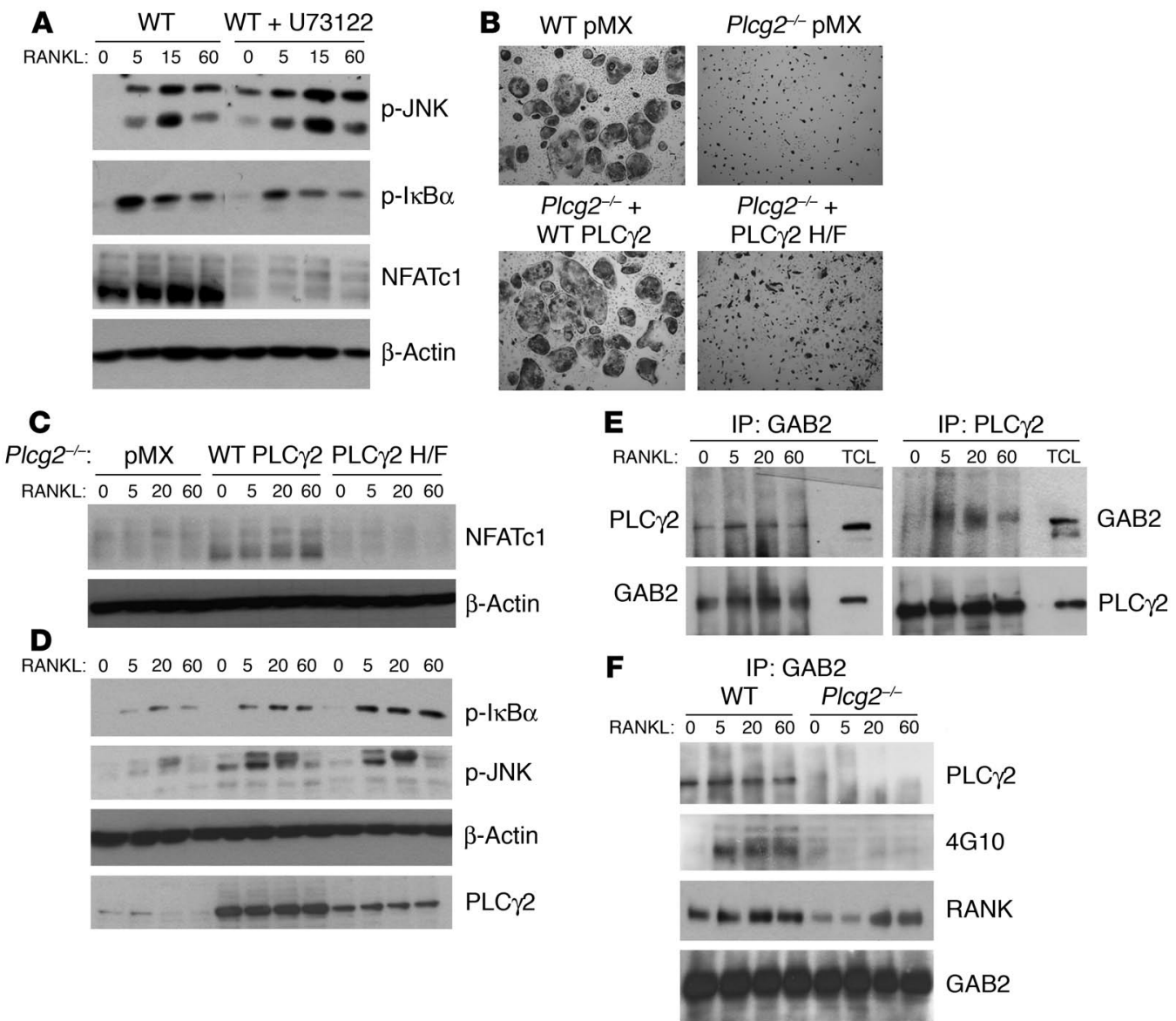

Figure 7

PLC $\gamma 2$ forms a complex with GAB2 and modulates its activation. (A) WT preOCs cultured with or without the PLC inhibitor U73122 (5 $\mu$ M) for 3 days were stimulated with RANKL and subjected to Western blot analysis for phospho-JNK, phospho-IKB $\alpha$, and NFATc1. $\beta$-Actin served as control. (B) WT and Plcg2 ${ }^{-1-}$ BMMs retrovirally transduced with empty vector (pMX), WT PLC $\gamma 2$, or catalytically inactive PLC $\gamma 2$ (PLC $\left.\gamma 2 \mathrm{H} / \mathrm{F}\right)$ were cultured with RANKL $(100 \mathrm{ng} / \mathrm{ml})$ and M-CSF $(10 \mathrm{ng} / \mathrm{ml})$ for 7 days, and multinucleated OCs were detected by TRAP staining. Objective, $\times 10$. (C) The same cells as shown in B were subjected to RANKL stimulation and Western blot analysis to detect NFATc1 expression. (D) PIcg2-/- BMMs retrovirally transduced with pMX, WT PLC $\gamma 2$, or PLC $\gamma 2 \mathrm{H} / \mathrm{F}$ were treated with RANKL, and phosphorylation of IKB $\alpha$ and JNK was determined by Western blot analysis. PLC $\gamma 2$ expression levels are shown. $\beta$-Actin served as loading control in $\mathbf{C}$ and $\mathbf{D}$. (E) PLC $\gamma 2$ and GAB2 were reciprocally immunoprecipitated in WT and Plcg2--- BMMs treated with RANKL and subjected to Western blot analysis using anti-PLC $\gamma 2$ and anti-GAB2 Abs, respectively. TCL, total cell lysate. (F) GAB2 was immunoprecipitated in WT and Plcg2-- BMMs and subjected to Western blot analysis using anti-phosphotyrosine Ab (clone 4G10), anti-RANK, and anti-PLC 2.

phosphorylated by RANKL. This result suggests that the 2 PLC $\gamma$ isoforms exert different, nonoverlapping functions in OCs.

Differential regulation of the 2 PLC $\gamma$ isoforms within the same cell type has been previously demonstrated in platelets, where activation of glycoprotein VI by collagen resulted in a substantial increase in tyrosine phosphorylation of PLC $\gamma 2$ but not PLC $\gamma 1$ (41, 42). Since activation of PLC $\gamma$ isoforms is tightly regulated by the action of a number of receptor tyrosine kinases (RTKs) (24-26), we asked whether M-CSF, via its receptor c-Fms, could lead to phosphorylation of PLC $\gamma 1$, PLC $\gamma 2$, or both. We found that PLC $\gamma 1$, and to a lesser extent PLC $\gamma 2$, was phosphorylated by M-CSF. In support of this finding, analysis of $\mathrm{Plcg}^{-1-}$ ES cells generated few hematopoietic cells with a reduced level of monocyte/macrophage differentiation (29). Furthermore, we did not detect an M-CSFdependent proliferative defect in BMMs and OCs lacking PLC $\gamma 2$, and we observed that M-CSF-mediated activation of ERK and JNK occurred normally. These results indicate that PLC $\gamma 2$ is not involved in M-CSF signaling and suggest that PLC $\gamma 1$ may regulate this pathway in OC lineage cells.

The phosphorylation of PLC $\gamma 2$ in response to RANKL was mediated in an SFK-dependent manner. PP2, a well-known Src kinase inhibitor, completely blocked RANKL-mediated PLC $\gamma 2$ activation. Moreover Dap12 was required for phosphorylation of PLC $\gamma 2$ in response to RANKL stimulation. It is possible that SFKs phosphorylate PLC $\gamma 2$ directly. However, it is more likely that SFKs phosphorylate the tyrosine residues in the ITAM motif of Dap12, leading to recruitment and activation of Syk, which in turn can activate PLC $\gamma$, as occurs in other cell types (B cells and mast cells) $(43,44)$. We did not determine which Src family member is involved in PLC $\gamma 2$ activation, since the inhibitor used blocked various SFKs. These results indicate that PLC $\gamma$ isoforms are differentially phosphorylated in OCs, PLC $\gamma 2$ primarily by RANKL and PLC $\gamma 1$ by M-CSF.

AP1, NF-кB, and NFATc1 are members of well-described osteoclastogenic pathways activated by RANKL. We found that PLC $\gamma 2$ 

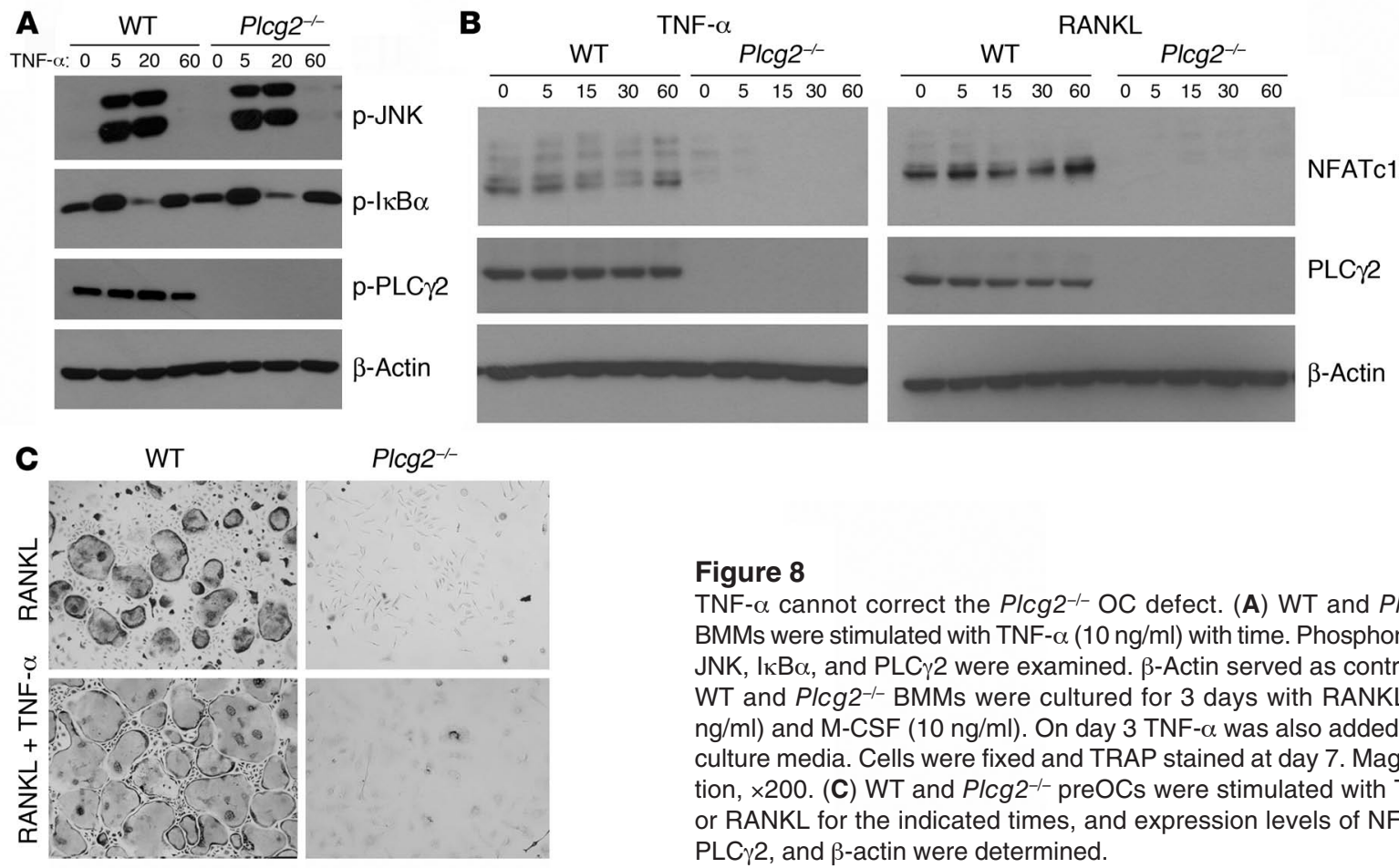

\section{Figure 8}

TNF- $\alpha$ cannot correct the Plcg2 $2^{-/-}$OC defect. (A) WT and Plcg2-/BMMs were stimulated with TNF- $\alpha(10 \mathrm{ng} / \mathrm{ml})$ with time. Phosphorylated $\mathrm{JNK}$, IKB $\alpha$, and PLC $\gamma 2$ were examined. $\beta$-Actin served as control. (B) WT and Plcg2-/- BMMs were cultured for 3 days with RANKL (100 $\mathrm{ng} / \mathrm{ml})$ and M-CSF (10 $\mathrm{ng} / \mathrm{ml})$. On day 3 TNF- $\alpha$ was also added to the culture media. Cells were fixed and TRAP stained at day 7. Magnification, $\times 200$. (C) WT and $\mathrm{Plcg}^{-1-}$ preOCs were stimulated with TNF- $\alpha$ or RANKL for the indicated times, and expression levels of NFATc1, PLC $\gamma 2$, and $\beta$-actin were determined.

differentially regulates the activation of all them. On one hand, inhibition of PLC $\gamma 2$ catalytic activity by U73122 completely abrogated NFATc1 expression in osteoclastic cells but had no effect on activation of JNK or phosphorylation of IкB $\alpha$. On the other hand, we found that p65 translocation into the nucleus and consequent NF- $\kappa \mathrm{B}$ activation determined by EMSA were virtually absent in PLC $\gamma 2$-deficient BMMs. Similarly, phospho-JNK levels, nuclear translocation of phospho-c-Jun, and thus activation of AP1 were also aberrant in the null cells. Therefore, these data suggest PLC $\gamma 2$ catalytic activity is required for NFATc1 expression but not for JNK and I $\mathrm{B} \alpha$ phosphorylation. In agreement with this hypothesis, a catalytically inactive mutant of PLC $\gamma 2$ could rescue neither the osteoclastogenic defect of cells lacking PLC $\gamma 2$ nor NFATc1 upregulation, while being sufficient to promote JNK and ІкB $\alpha$ phosphorylation in response to RANKL. Interestingly, the finding that PLC $\gamma 2$ is activated by RANKL downstream of Dap 12 and governs all the above-mentioned signaling pathways differs from the data obtained with cells lacking Dap12 and FcR $\gamma$. In fact, Dap12-/$F c R \gamma^{-/}$OCs displayed normal RANKL-mediated phosphorylation of JNK, c-Jun, and IкB $\alpha$, while NFATc1 upregulation was absent (20). These results suggest that PLC $\gamma 2$ may not be uniquely involved in the signaling cascade downstream of Dap12/FcR $\gamma$ receptor and in fact indicate that PLC $\gamma 2$ regulates JNK and I $\mathrm{B} \alpha \alpha$ phosphorylation downstream of RANKL independent of Dap12/FcR $\gamma$.

The adapter protein GAB2 has been shown to be a crucial component of RANK signaling and osteoclastogenesis and is required for activation of NF-KB and JNK, but not NFATc1, pathways (16). Based on the observation that PLC $\gamma 2$ forms a complex with GAB2 in OCs and in other cell types (36), we hypothesized that PLC $\gamma 2$ could regulate some RANKL signaling pathways by acting as an adapter molecule. One possibility is that PLC $\gamma 2$ modulates the GAB2/RANK complex. In support of this hypothesis, we found that PLC $\gamma 2$ is required for GAB2 phosphorylation and mediates
GAB2 recruitment to RANK, suggesting that AP1 and NF- $\mathrm{B}$ activation might be dependent on the capacity of PLC $\gamma 2$ to form a complex with GAB2. In addition, the finding that the PLC inhibitor U73122, which blocks IP3-mediated calcium influx (45), and a catalytically inactive form of PLC $\gamma 2$ completely abrogate osteoclastogenesis by affecting NFATc1 upregulation suggest that both the catalytic activity and the adapter function of PLC $\gamma 2$ are required for osteoclastogenesis. Thus, overall our data support the model (Figure 9) in which the catalytic activity of PLC $\gamma 2$ is required for NFATc1 upregulation downstream of the ITAM motif of Dap12, while PLC $\gamma 2$, acting as an adapter molecule, mediates GAB2 phosphorylation and its recruitment to RANK, leading to AP1 and NF- $\mathrm{KB}$ activation.

The specific requirement of PLC $\gamma 2$ for activation of NF- $\mathrm{KB}, \mathrm{AP} 1$, and NFATc1 signaling pathways is manifested in a basal in vivo osteopetrotic phenotype characterized by defective OC recruitment. Although the number of OCs in vivo was significantly reduced in Plcg2-null compared with WT animals, the presence of a few TRAP-positive cells could reflect the presence of a milieu of cytokines circulating in vivo that partially rescue the differentiation of PLC $\gamma 2$-null OCs. TNF- $\alpha$ potently stimulates osteoclastogenesis by augmenting RANKL-mediated signaling pathways (46). TNF- $\alpha$

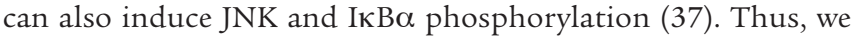
sought to determine whether TNF- $\alpha$ could reverse the ability of the PLC $\gamma 2$-null cells to become OCs. Interestingly, while TNF- $\alpha$ could activate JNK and IאB $\alpha$ in $P l c g 2^{-/-}$BMMs, it could not reverse their differentiation defect, most likely due to the inability of TNF- $\alpha$ to induce NFATc1 upregulation. In conclusion, our data indicate that PLC $\gamma 2$ is required for the maintenance of bone homeostasis and RANKL-mediated osteoclastogenesis both in vitro and in vivo.

Previous studies reported that $P l c g 2^{-/}$mice have defective B cell maturation and mast cell activation (29). The inability of Plcg2-/- cells to respond to both basal and inflammation-mediated 


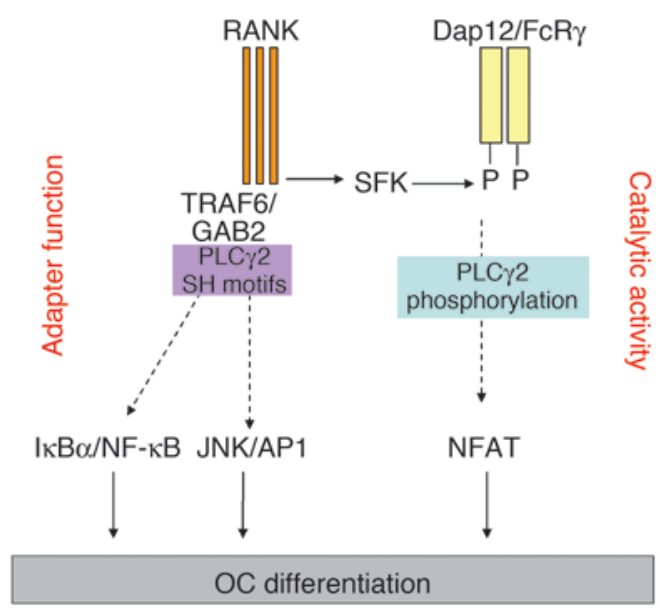

Figure 9

PLC $\gamma 2$ in RANKL signaling. PLC $\gamma 2$ is phosphorylated by RANKL in an SFK-dependent manner downstream of Dap12/FcR $\gamma$. Activation of PLC $\gamma 2$ and PLC $\gamma 2$ catalytic activity are required for NFATc1 upregulation (right arm). PLC $\gamma 2$ can also bind GAB2 and modulate its activation and recruitment to the RANK-signaling complex (left arm). This interaction might be required for activation of the $\mathrm{I}_{\kappa} \mathrm{B} \alpha / \mathrm{NF}-\kappa \mathrm{K}$ and JNK/AP1 pathways independent of Dap12/FcR $\gamma$.

osteoclastogenesis in vitro clearly supports the important role of PLC $\gamma 2$ in osteoclast differentiation. Thus, the intrinsic defect of Plcg2 $2^{--}$OCs together with the deficient B cell-mediated immune response suggest that targeting PLC $\gamma 2$ could be a novel approach for antiresorptive therapies. Antirheumatic therapies directed to selectively deplete human CD20-positive B cells (e.g., Rituximab) or to directly target OC activation and function (RANKL inhibitors and bisphosphonates) have been very useful approaches in recent clinical trials (47). However, in most cases, only a combination of both treatments will resolve both the inflammatory and the ongoing osteolytic responses. Thus, PLC $\gamma 2$ becomes an interesting target for the cure of rheumatoid diseases due to its dual role in the activation of a particular subset of immune cells, B lymphocytes, and for its capacity to control OC development in vivo. Therefore, a combined therapy affecting both the inflammatory and the osteolytic components of the rheumatoid disease might increase the hope that disease modification and remission in rheumatoid arthritis is a realistic goal.

\section{Methods}

Mice. Plcg2 $2^{-/-}$, Dap12 $2^{-/-}$FcR $\gamma^{-/-}$, and WT mice were on a C57BL/6 background and have been previously described $(20,29)$. All mice used in these experiments were 6-16 weeks old and bred and maintained with sterilized food, water, and bedding at the Animal Facility of the Washington University School of Medicine. All experiments were approved by the Animal Studies Committee of Washington University.

Histology and $\mu C T$. Five-micron sections of fixed, decalcified, paraffinembedded long bones were stained with either $\mathrm{H} \& \mathrm{E}$ or histochemically for TRAP to detect OCs. Calvariae from mice injected with calcein $(20 \mathrm{mg} / \mathrm{kg}$ dissolved in $2 \% \mathrm{NaHCO}_{3}$; Sigma-Aldrich) were embedded in plastic. For $\mu \mathrm{CT}, 3 \mathrm{D}$ images from intact mouse femurs were obtained on a $\mu \mathrm{CT} 40$ scanner (Scanco Medical).

Mouse OCs. BMMs were isolated from long bones of 6-to 8-week-old mice as described previously (48). We obtained OCs by culturing BMMs with
glutathione-S-transferase-RANKL (GST-RANKL) $(100 \mathrm{ng} / \mathrm{ml})$ and M-CSF $(10 \mathrm{ng} / \mathrm{ml})$ for 5 days. For coculture experiments, OBs and BMMs were mixed and cultured for $10-14$ days in the presence of 1,25 Vit $\mathrm{D}_{3}\left(10^{-8} \mathrm{M}\right)$. For rescue experiments, macrophages were obtained as described above; transduced with Flag-Dap12, Flag-PLC $\gamma 2$, or Flag-PLC $\gamma 2 \mathrm{H} / \mathrm{F}$; selected in puromycin- (for Dap12 constructs) or blastocydin-containing media (for PLC $\gamma 2$ constructs) for 3 days; and cultured with M-CSF and RANKL to obtain OCs, as previously described (49). The PLC $\gamma 2 \mathrm{H} / \mathrm{F}$ mutation was made using a QuikChange XL Site-Directed Mutagenesis Kit (catalog 200517; Stratagene) following the manufacturer's instructions.

Mouse OBs. We isolated OB precursors from calvariae of mice that were 4 days old, expanded them in media alone until confluent, and then cultured them with $\beta$-glycerolphosphate and ascorbic acid to induce OB differentiation until bone nodules formed.

Western blot analysis and antibodies. BMMs or OCs starved for 6 hours in serum-free medium were stimulated with RANKL $(100 \mathrm{ng} / \mathrm{ml})$ or M-CSF $(100 \mathrm{ng} / \mathrm{ml})$ and lysed at the times indicated in the Results and Figures 4-8 in RIPA lysis buffer supplemented with protease inhibitors and sodium orthovanadate. In some experiments, OCs were cultured in the presence of the Src inhibitor PP2 $(5 \mu \mathrm{M}$; Calbiochem $)$ or PLC inhibitor U73122 (5 $\mu \mathrm{M}$; BIOMOL), starved, and then stimulated with RANKL. For Western blot analysis of phospho-PLC $\gamma 1$ and -PLC $\gamma 2$, phospho-ERK, phospho-JNK, phospho-c-Jun, phospho-IкB $\alpha$, and phospho-Src (Y416), we used polyclonal antibodies from Cell Signaling Technology. Polyclonal antiserum against p65, NFATc1, and PLC $\gamma 2$ were purchased from Santa Cruz Biotechnology Inc. The monoclonal antibody against $\beta$-actin was obtained from Sigma-Aldrich. Polyclonal GAB2 Ab was purchased from Upstate USA Inc. To obtain nuclear extract from RANKL-treated cells, plates were washed with $\mathrm{H}_{2} \mathrm{O}$ and cells lysed with hypotonic buffer (10 mM HEPES, $1.5 \mathrm{mM} \mathrm{MgCl}_{2}, 1 \mathrm{mM} \mathrm{KCl}, 1 \mathrm{mM}$ DTT, and protease and phosphatase inhibitors), followed by addition of $0.1 \%$ NP40. After centrifugation, the supernatants were collected (cytosolic fraction), while the pellets (nuclear fraction) were suspended in high-salt buffer (hypotonic buffer plus $400 \mathrm{mM} \mathrm{NaCl}$ ). Extracts were quantified by a modified Coomassie method (Pierce). Nonradioactive EMSA was performed following the manufacturer's instructions (Pierce) using AP1 and NF- $\kappa B$ biotinylated oligonucleotides (for AP1: 5' Biotin-ACGCTTGATGACTCAGCCGGAAT-3' and 5' Biotin-ATTCCGGCTGAGTCATCAAGC-3'; for NF-кB: $5^{\prime}$ Biotin-AAGTTGAGGGGACTTTCCCAGGCT-3' and 5' Biotin-AGCCTGGGAAAGTCCCCTCAACTT-3'). An excess (200x) of nonbiotinylated oligonucleotides was used for competition control.

Immunofluorescence. WT or Dap $12^{-/-} F c R \gamma^{-/-}$OCs retrovirally transduced with either the empty vector or full-length Dap12 were cultured on glass, fixed, and stained with anti-NFATc1 Ab (Santa Cruz Biotechnology Inc.), DAPI to detect the nuclei, and FITC-phalloidin to detect actin (48).

Real-time PCR. Total RNA was isolated from cell cultures at various times using the RNeasy Mini Kit (QIAGEN) and was reverse transcribed to cDNAs using SuperScript II according to the manufacturer's instructions (Invitrogen). Primers specific for murine NFATc1, TRAP, GAPDH, cathepsin $\mathrm{K}$, and calcitonin receptor were used. For quantitative real-time PCR, NFATc1 was amplified using 5'-CCCGTCACATTCTGGTCCAT-3' and $5^{\prime}$-CAAGTAACCGTGTAGCTGCACAA-3'.

TRAP was amplified using $5^{\prime}$-CAGCTCCCTAGAAGATGGATTCAT$3^{\prime}$ and $5^{\prime}$-GTCAGGAGTGGGAGCCATATG- $3^{\prime}$; $\beta$-actin using $5^{\prime}$-CTTCTACAATGAGCTGCGTG- $3^{\prime}$ and $5^{\prime}$-TCATGAGGTAGTCTGTCAGG$3^{\prime}$; cathepsin $\mathrm{K}$ using $5^{\prime}$-ATGTGGGTGTTCAAGTTTCTGC-3' and $5^{\prime}$-CCACAAGATTCTGGGGACTC-3'; and calcitonin receptor using 5'-CAAGAACCTTAGCTGCCAGAG-3' and 5'-CAAGCACGCGGACAATGTTG-3'. SYBR green dye was used for detection of the product using the SYBR Green PCR Master Mix assay (Applied Biosystems). The standard 
curve used a series of duplicate dilutions of plasmid for each gene and $\beta$-actin cDNA. The amplification reaction was performed for 40 cycles with denaturation at $95^{\circ} \mathrm{C}$ for 10 minutes, followed by annealing at $95^{\circ} \mathrm{C}$ for 15 seconds and extension and detection at $60^{\circ} \mathrm{C}$ for 1 minute. The relative RNA abundance of each target gene transcript was normalized against endogenous gene control and calculated as $2^{-\Delta c t(t a r g e t ~ g e n e-~ e n d o g e n o u s ~ c o n t r o l ~ g e n e), ~}$ where ct represents the threshold cycle for each transcript.

Statistics. All data are presented as mean \pm SEM. Values with $P<0.05$ were considered significant as determined by 2 -tailed Student's $t$ test (SigmaPlot version 8; Systat).

\section{Acknowledgments}

We gratefully acknowledge Yousef Abu-Amer (Department of Orthopaedic Surgery, Washington University) and Marco Colonna (Department of Pathology and Immunology, Washington University) for helpful suggestions and discussion; James N. Ihle for Plcg $2^{-/-}$mice (St. Jude Children's Research Hospital, Memphis,
Tennessee, USA); and Matilda Katan for the full-length PLC $\gamma 2$ construct (Cancer Research UK Centre for Cell and Molecular Biology, Chester Beatty Laboratories). This work was supported by departmental funding from the Orthopaedic Surgery Department, Washington University School of Medicine; by the Arthritis Foundation (R. Faccio); by an American Society for Bone and Mineral Research career award (to R. Faccio); and by NIH grants (AR47846 and AR48335 to D.V. Novack and AR52921 to R. Faccio).

Received for publication April 7, 2006, and accepted in revised form August 29, 2006.

Address correspondence to: Roberta Faccio, Washington University School of Medicine, Department of Orthopaedic Surgery, Campus Box 8233, 1 Barnes Jewish Hospital Plaza, Suite 11300, St. Louis, Missouri 63110, USA. Phone: (314) 747-4602; Fax: (314) 362-0334; E-mail: faccior@wustl.edu.
1. Goldring, S.R., and Gravallese, E.M. 2000. Mechanisms of bone loss in inflammatory arthritis: diagnosis and therapeutic implications. Arthritis Res. 2:33-37.

2. Teitelbaum, S.L., and Ross, F.P. 2003. Genetic regulation of osteoclast development and function. Nat. Rev. Genet. 4:638-649.

3. Zhang, Y.-H., Heulsmann, A., Tondravi, M.M., Mukherjee, A., and Abu-Amer, Y. 2001. Tumor necrosis factor-alpha (TNF) stimulates RANKLinduced osteoclastogenesis via coupling of TNF type 1 receptor and RANK signaling pathways. J. Biol. Chem. 276:563-568.

4. Kitaura, H., et al. 2005. M-CSF mediates TNFinduced inflammatory osteolysis. J. Clin. Invest. 115:3418-3427. doi:10.1172/JCI26132.

5. O' Gradaigh, D., Ireland, D., Bord, S., and Compston, J.E. 2004. Joint erosion in rheumatoid arthritis: interactions between tumour necrosis factor alpha, interleukin 1 , and receptor activator of nuclear factor kappaB ligand (RANKL) regulate osteoclasts. Ann. Rheum. Dis. 63:354-359.

6. Wei, S., Kitaura, H., Zhou, P., Ross, F.P., and Teitelbaum, S.L. 2005. IL-1 mediates TNF-induced osteoclastogenesis. J. Clin. Invest. 115:282-290. doi:10.1172/JCI200523394

7. Tanaka, S., Nakamura, K., Takahasi, N., and Suda, T. 2005. Role of RANKL in physiological and pathological bone resorption and therapeutics targeting the RANKL-RANK signaling system. Immunol. Rev. 208:30-49.

8. Karin, M., Cao, Y., Greten, F.R., and Li, Z.-W. 2002. NF-kappaB in cancer: from innocent bystander to major culprit. Nat. Rev. Cancer. 2:301-310.

9. David, J.P., Sabapathy, K., Hoffmann, O., Idarraga, M.H., and Wagner, E.F. 2002. JNK1 modulates osteoclastogenesis through both c-Jun phosphorylation-dependent and -independent mechanisms. J. Cell Sci. 115:4317-4325.

10. Ruocco, M.G., et al. 2005. IkappaB kinase (IKK)beta, but not IKKalpha, is a critical mediator of osteoclast survival and is required for inflammationinduced bone loss. J. Exp. Med. 201:1677-1687.

11. Dai, S., Hirayama, T., Abbas, S., and Abu-Amer, Y. 2004. The IkappaB kinase (IKK) inhibitor, NEMObinding domain peptide, blocks osteoclastogenesis and bone erosion in inflammatory arthritis. J. Biol. Chem. 279:37219-37222.

12. Jimi, E., et al. 2004. Selective inhibition of NFkappaB blocks osteoclastogenesis and prevents inflammatory bone destruction in vivo. Nat. Med. 10:617-624.

13. Iotsova, V., et al. 1997. Osteopetrosis in mice lacking NF-kappaB1 and NF-kappaB2. Nat. Med. 3:1285-1289.
14. Franzoso, G., et al. 1997. Requirement for NFkappaB in osteoclast and B-cell development. Genes Dev. 11:3482-3496.

15. Grigoriadis, A.E., et al. 1994. c-Fos: a key regulator of osteoclast-macrophage lineage determination and bone remodeling. Science. 266:443-448.

16. Wada, T., et al. 2005. The molecular scaffold Gab2 is a crucial component of RANK signaling and osteoclastogenesis. Nat. Med. 11:394-399.

17. Asagiri, M., et al. 2005. Autoamplification of NFATc1 expression determines its essential role in bone homeostasis. J. Exp. Med. 202:1261-1269.

18. Takayanagi, H., et al. 2002. Induction and activation of the transcription factor NFATc1 (NFAT2) integrate RANKL signaling in terminal differentiation of osteoclasts. Dev. Cell. 3:889-901.

19. Ikeda, F., et al. 2004. Critical roles of c-Jun signaling in regulation of NFAT family and RANKLregulated osteoclast differentiation. J. Clin. Invest. 114:475-484. doi:10.1172/JCI200419657.

20. Koga, T., et al. 2004. Costimulatory signals mediated by the ITAM motif cooperate with RANKL for bone homeostasis. Nature. 428:758-763.

21. Kaifu, T., et al. 2003. Osteopetrosis and thalamic hypomyelinosis with synaptic degeneration in DAP12-deficient mice. J. Clin. Invest. 111:323-332. doi:10.1172/JCI200316923.

22. Mocsai, A., et al. 2004. The immunomodulatory adapter proteins DAP12 and Fc receptor gammachain (FcRgamma) regulate development of functional osteoclasts through the Syk tyrosine kinase. Proc. Natl. Acad. Sci. U. S. A. 101:6158-6163.

23. Humphrey, M.B., et al. 2004. The signaling adapter protein DAP12 regulates multinucleation during osteoclast development. J. Bone Miner. Res. 19:224-234.

24. Wilde, J.I., and Watson, S.P. 2001. Regulation of phospholipase C gamma isoforms in haematopoietic cells: why one, not the other? Cell. Signal. 13:691-701.

25. Rebecchi, M.J., and Pentyala, S.N. 2000. Structure, function, and control of phosphoinositide-specific phospholipase C. Physiol. Rev. 80:1291-1335.

26. Katan, M. 1998. Families of phosphoinositidespecific phospholipase C: structure and function. Biochim. Biophys. Acta. 1436:5-17.

27. Katan, M. 2005. New insights into the families of PLC enzymes: looking back and going forward. Biochem. J. 391:e7-e9.

28. Ji, Q.S., et al. 1997. Essential role of the tyrosine kinase substrate phospholipase C-gamma1 in mammalian growth and development. Proc. Natl. Acad. Sci. U. S. A. 94:2999-3003.

29. Wang, D., et al. 2000. Phospholipase Cgamma2 is essential in the functions of $\mathrm{B}$ cell and several FC receptors. Immunity. 13:25-35.

30. Hashimoto, A., et al. 2000. Cutting edge: essential role of phospholipase C-gamma 2 in B cell development and function. J. Immunol. 165:1738-1742.

31. Tassi, I., et al. 2005. Phospholipase C-gamma 2 is a critical signaling mediator for murine NK cell activating receptors. J. Immunol. 175:749-754.

32. Takayanagi, H., et al. 2000. Involvement of receptor activator of nuclear factor kappaB ligand/osteoclast differentiation factor in osteoclastogenesis from synoviocytes in rheumatoid arthritis. Arthritis Rheum. 43:259-269.

33. Antony, P., et al. 2004. B-cell antigen receptor activates transcription factors NFAT (nuclear factor of activated T-cells) and NF-kappaB (nuclear factor kappaB) via a mechanism that involves diacylglycerol. Biochem. Soc. Trans. 32:113-115.

34. Faccio, R., Zou, W., Colaianni, G., Teitelbaum, S.L., and Ross, F.P. 2003. High dose M-CSF partially rescues the Dap12-/- osteoclast phenotype. J. Cell. Biochem. 90:871-883.

35. Smith, M.R., et al. 1994. Phospholipase C-gamma 1 can induce DNA synthesis by a mechanism independent of its lipase activity. Proc. Natl. Acad. Sci. U. S. A. 91:6554-6558.

36. Xie, Z.H., Ambudkar, I., and Siraganian, R.P. 2002. The adapter molecule Gab2 regulates Fc epsilon RI-mediated signal transduction in mast cells. J. Immunol. 168:4682-4691.

37. Wei, S., Wang, M.W., Teitelbaum, S.L., and Ross, F.P. 2002. Interleukin-4 reversibly inhibits osteoclastogenesis via inhibition of NF-KB and MAP kinase signaling. J. Biol. Chem. 21:6622-6630.

38. Arron, J.R., and Choi, Y. 2000. Bone versus immune system. Nature. 408:535-536.

39. Wiebe, S.H., Hafezi, M., Sandhu, H.S., Sims, S.M., and Dixon, S.J. 1996. Osteoclast activation in inflammatory periodontal diseases. Oral Dis. 2:167-180.

40. Yu, P., et al. 2005. Autoimmunity and inflammation due to a gain-of-function mutation in phospholipase C gamma 2 that specifically increases external Ca2+ entry. Immunity. 22:451-465.

41. Blake, R.A., Asselin, J., Walker, T., and Watson, S.P. 1994. Fc gamma receptor II stimulated formation of inositol phosphates in human platelets is blocked by tyrosine kinase inhibitors and associated with tyrosine phosphorylation of the receptor. FEBS Lett. 342:15-18.

42. Daniel, J.L., Dangelmaier, C., and Smith, J.B. 1994. Evidence for a role for tyrosine phosphorylation of phospholipase $\mathrm{C}$ gamma 2 in collagen-induced platelet cytosolic calcium mobilization. Biochem. J. 302:617-622.

43. Tamir, I., and Cambier, J.C. 1998. Antigen receptor 
signaling: integration of protein tyrosine kinase functions. Oncogene. 17:1353-1364.

44. Sada, K., and Yamamura, H. 2003. Protein-tyrosine kinases and adaptor proteins in FcepsilonRI-mediated signaling in mast cells. Curr. Mol. Med. 3:85-94. 45. Bleasdale, J.E., et al. 1989. Inhibition of phospholipase $\mathrm{C}$ dependent processes by U-73, 122. Adv . Prostaglandin Thromboxane Leukot. Res. 19:590-593.
46. Lam, J., et al. 2000. TNF- $\alpha$ induces osteoclastogenesis by direct stimulation of macrophages exposed to permissive levels of RANK ligand. J. Clin. Invest. 106:1481-1488.

47. Genovese, M.C. 2005. Biologic therapies in clinical development for the treatment of rheumatoid arthritis. J. Clin. Rheumatol. 11:S45-S54.

48. Faccio, R., Novack, D.V., Zallone, A., Ross, F.P., and Teitelbaum, S.L. 2003. Dynamic changes in the osteoclast cytoskeleton in response to growth factors and cell attachment are controlled by b3 integrin. J. Cell Biol. 162:499-509.

49. Faccio, R., Zallone, A., Ross, F.P., and Teitelbaum, S.L. 2003. c-Fms and the avb3 integrin collaborate during osteoclast differentiation. J. Clin. Invest. 111:749-758. doi:10.1172/JCI200316924. 